\title{
Conductance measurement of a conical tube and calculation of the pressure distribution
}

\author{
B. Mercier ${ }^{a)}$ \\ Laboratoire de l'Accélérateur Linéaire (LAL), Université Paris-Sud, Batiment 200, BP34, \\ 91898 Orsay Cedex, France
}

()

In recent articles [J. Gomez-Goni, and P. J. Lobo J. Vac. Sci. Technol. A 21, 1452 (2003); P. Swemin and M. Niewinski, Vacuum 67, 359 (2002)] the conductance of conical tube in the molecular flow regime has been calculated using the Monte Carlo method or by the resolution of the Clausing integral equation, reformulated by Iczkowski et al. [J. Phys. Chem. 67, 229 (1963)], for the case of a cone. The comparison between the analytical values and different simulations allows one to determine a correction factor $k$ to apply to the intrinsic conductance of the cones. This coefficient depends on the propagation direction of flow and increases considerably for larger conic angles. For a cone half-angle of $40^{\circ}$ and a length ten times greater than smallest entrance radii, the correction factor is approximately 5.3 for a circulating flow from the smallest to the largest orifice. Our experimental device measured the conical conductance by a dynamic method. In order to do this, it was necessary to determine the surface pressure distribution. The extension of the Oatley method, with the addition of several components of various transmission probabilities, permits one to establish this distribution for a vacuum system and thus to give the pressure measured by a gauge situated along the wall of the duct. This method provides a good approximation for tubes and cones and can be used for engineering practice. The determination of this distribution is all the more critical when the conductance and the pumping speed are large and can thus have a great influence on the vacuum metrology.

[]

\section{INTRODUCTION}

The first analytical calculations of conductance were carried out by Knudsen using the following expression:

$$
C_{L}=\frac{1}{4} V_{m} S W \quad \text { with } W=\frac{16}{3} \frac{S}{U L},
$$

where $V_{m}$ represents the average velocity of the molecules for a Maxwell-Boltzmann distribution, $S$ the cross sectional area of the inlet aperture, $U$ the perimeter, and $L$ the length of the duct. However, this formula is valid only for infinitely long tubes compared to their diameters. Smoluchowski ${ }^{1}$ showed that this equation is incorrect and that it is necessary to replace it by

$$
C_{L}=\frac{1}{4} V_{m} \frac{A}{2 L} \quad \text { with } A=\int_{S} \int_{-\pi / 2}^{+\pi / 2} \frac{1}{2} \rho^{2} \cos (\theta) d \theta d s,
$$

where $\rho$ is a cord of the section making an angle $\theta$ with the normal to the perimeter $s$. The resolution of the integral introduces a correction factor $k$ into Knudsen's formula,

$$
W=\frac{16}{3} \frac{S}{U} \frac{k}{L}
$$

This coefficient $k$ takes into account the different geometry of the inlet aperture. This expression remains valid for an infinitely long tube conductance. To take into account a short

\footnotetext{
a) Author to whom correspondence should be addressed; electronic mail: mercier@1al.in2p3.fr
}

tube conductance, Dushman combines $C_{L}$ with an entrance conductance $C_{e}$ (for $L \rightarrow 0$ then $C \rightarrow C_{e}$ ).

$$
C=\frac{C_{L} C_{e}}{C_{L}+C_{e}} .
$$

In the case of an infinitely large upstream chamber, $C_{e}$ $=1 / 4 V_{m} S$. Many references ${ }^{1,2}$ give the expression for the conductance value for sections which vary with their lengths, by integrating Knudsen's equation.

$$
C_{L}=\frac{4}{3} V_{m} \frac{1}{\int_{0}^{L}\left(U / k S^{2}\right) d l} .
$$

However, the equations above are only approximations and it is often necessary, when the geometrical forms become complex, to determine the conductance by the Monte Carlo method or by solving the numerical integral of Clausing. ${ }^{3}$ These two methods allow one to calculate the transmission probability of the molecules which corresponds to the factor $W$. For a cylindrical tube, the relative error between the Dushman equation and the Clausing equation reaches $12 \%$ and it can become higher than $50 \%$ for complex geometries.

\section{CALCULATION OF THE CONICAL CONDUCTANCE}

\section{A. Analytical calculation}

We consider a conical tube (Fig. 1) of length $L, R_{0}$, and $R_{k}$ being the smallest and largest radii, respectively, and $\alpha$ its half-angle. We distinguish two intrinsic conductances ac- 


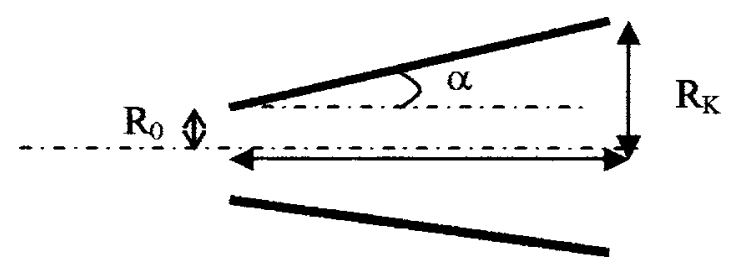

FIG. 1. Conical tube scheme.

cording to the flow direction. "Direct" conductance $C_{A}$ for flow circulating from $R_{0}$ towards $R_{k}$ and "back" conductance $C_{R}$ for flow in the opposite direction.

The integration of Eq. (4), with $k$ supposed to be constant for a given angle, is expressed by

$$
C_{A}=\frac{4}{3} \pi V_{m} \frac{R_{0}^{2} R_{K}^{2} k}{\left(R_{0}+R_{K}\right) L} .
$$

The conductance value $C_{R}$ can be calculated by applying the Dushman equation and the equilibrium condition of flows $1 / C_{R}+1 / C_{e, R}=1 / C_{A}+1 / C_{e, A}$, we obtain

$$
\gamma=\frac{C_{A}}{C_{R}}=1+\frac{16}{3} k \tan (\alpha)
$$

We will determine this correction factor $k$ by the Monte Carlo method.

\section{B. Monte Carlo method}

Gomez-Goni and Lobo ${ }^{4}$ and Swemin and Niewinski ${ }^{5}$ carried out a comparison between the Monte Carlo method and the numerical resolution of the Clausing integrals. These integrals were reformulated by Iczkowski ${ }^{6}$ for the case of the conical tube. This study made it possible to validate the method of resolution by obtaining very small relative variations. The transmission probability by Monte Carlo method is calculated with the following assumptions: (1) the flow must be molecular, (2) the adsorption of molecules on the chamber is neglected, (3) the law of emission of the molecules is assumed to follow a cosine distribution (Lambert's law), and (4) collisions with the walls are subject to diffuse reflection (cosine law). Assumption (3) supposes that the upstream chamber is infinitely large and that its speed distribution is of the Maxwell-Boltzmann type. Under these conditions,

$$
C_{M}=\frac{1}{4} V_{m} S W_{M}=C_{e} W_{M} .
$$

$W_{m}$ represents the transmission probability of the molecules calculated by the Monte Carlo method. According to Eqs. (5) and (7), and by taking account of the entrance conductance, we can express the correction factor $k$ as a function of $W$. For the case of the direct cone, we get

$$
\frac{1}{C_{M, A}}=\frac{1}{C_{e, A}}+\frac{1}{C_{A}}
$$

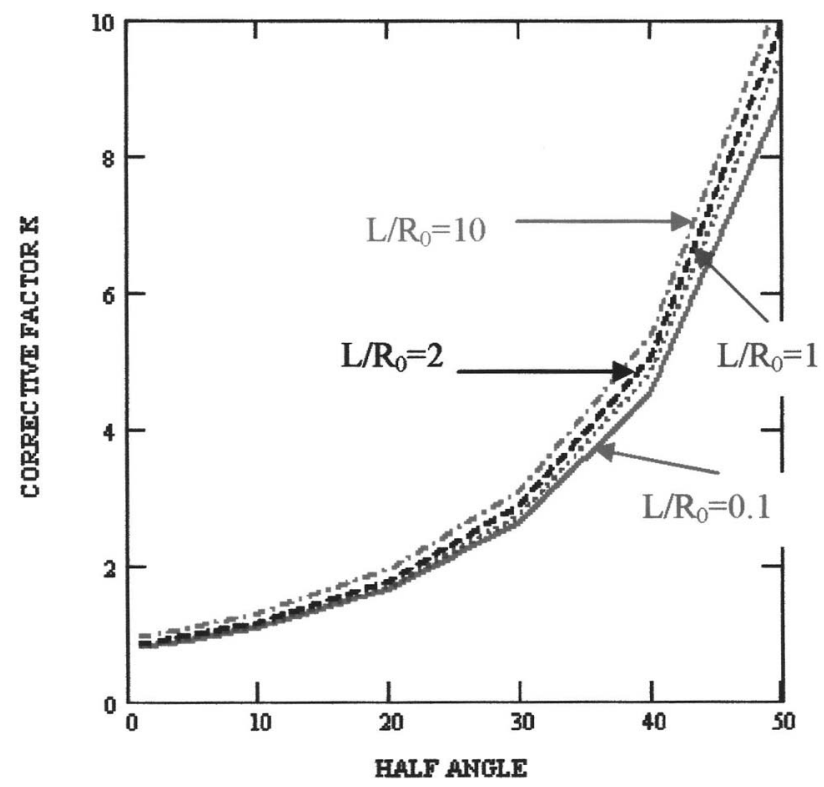

FIG. 2. Correction factor $k$ as function of half-angle of cone $\alpha$ and for different $L / R_{0}$.

$$
k=\frac{3}{16} \frac{W_{M, A}}{1-W_{M, A}} \frac{1}{R_{0} / L+\tan (\alpha)}\left[\frac{1}{1+\left(L / R_{0}\right) \tan (\alpha)}+1\right] .
$$

The values of $W_{M, A}$ were determined by various programs: a Monte Carlo (MC) program coming from CERN ${ }^{7}$ a direct simulation Monte Carlo (DSMC) program of Bird, ${ }^{8}$ and the data published in the article of Gomez-Goni and Lobo. ${ }^{4}$ According to Fig. 2, the correction factor $k$ becomes considerable for large angles and translates the fact that the reflection of the particles is diffuse compared to the normal of the wall surface and thus is a function of the cone slope. For example, a direct cone of ratio $L / R_{0}=10$ and angle $\alpha=40^{\circ}$ has a correction factor of approximately 5.3 and consequently the intrinsic backconductance of the cone will be approximately 25 times lower than that for the direct direction.

\section{EXPERIMENTAL MEASUREMENTS}

\section{A. Experimental device}

The measurement of the conductance is performed using a dynamic method. It consists of injecting a known flow $Q$, by the intermediary of a fine capillary of conductance $C_{1}$, and measuring pressure differential on both sides of the conductance $C_{R, 1}=Q /\left(P_{\mathrm{JAB} 1}-P_{\mathrm{JAB} 2}\right)$.

Figure 3 represents the experimental device, with an input flow line consisting of a chamber of volume $V$. This volume is maintained with a work pressure $P_{0}$ by an adjustable leak and this pressure is measured using capacitance diaphragm gauges (Baratron 627A MKS of 100 and 1 Torr full scale). This volume is connected to a fine capillary with a diameter of $0.8 \mathrm{~mm}$ and a length of $510 \mathrm{~mm}$. The dimensions of the cones $C_{R, 1}$ and $C_{A, 2}$ are expressed in millimeter and their half-angles are $40.28^{\circ}$ and $38.55^{\circ}$, respectively. Special at- 


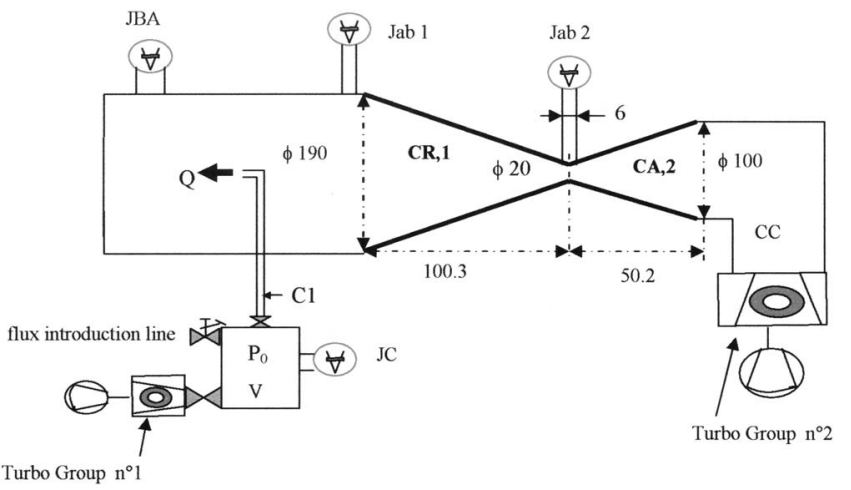

FIG. 3. Experimental device.

tention was paid so that the half-angle is constant over the entire length of the cones and so that the angles on the level of the junction of the two cones and the junction with the JAB2 gauge tube are sharp. The diameter of the junction of the two cones is $20 \mathrm{~mm} \pm 0.1 \mathrm{~mm}$. The measurement of the pressure is carried out using two identical spinning rotor gauges (Viscovac of Leybold). The second pumping installation is composed of a hybrid turbomolecular pump (TV141 Navigator of Varian), nominal pumping speed for nitrogen $S_{2}=125 \mathrm{l} \mathrm{s}^{-1}$, and a diaphragm pump Vacuubrand MZ2D of $1.9 \mathrm{~m}^{3} \mathrm{~h}^{-1}$. Later, a prepumping elbow, CC, was added. The intrinsic conductance was $359 \mathrm{1} \mathrm{s}^{-1}$ (unless specified, the values of conductance or flow are given for nitrogen at $\left.20{ }^{\circ} \mathrm{C}\right)$

\section{B. The measurement of flow}

The measurement of flow involves the use of the small capillary conductance; it is thus important to know its value. We used the method of the pressure evolution for the volume $V$. This method consists in measuring the time $\Delta t$ to pass from a pressure $P_{0}$ to $P_{0, t}$. The following assumptions are necessary; outgassing from the walls of the introduction chamber and from the capillary is negligible as compared to the introduced flow and it is considered that the temperature is constant. With the ideal gas law, we get

$$
V \frac{d P_{0}}{d t}=-P_{0} C_{1} .
$$

The conductance $C_{1}$ depends on the flow regime. The full curve, the circles, and crosses of Fig. 4(b) represent, respectively, the theoretical value given by Knudsen and the value measured by $C_{1}=(-V / P)(\Delta P /(\Delta t)$ by assuming a weak variation of $P$.

There is a good agreement between the measurement and the theory. The divergence noted while approaching the molecular mode can be explained by the modification of the equivalent length due to the capillary's elbow. In our case, to make precision measurements, it is necessary that the pressures measured by the spinning rotor gauges are higher than $5 \times 10^{-6}$ mbar. For that, in comparison with the conductance value of the prepumping elbow, CC, nominal pumping speed $S_{2}$ and conductance $C_{R, 1}=35.31 \mathrm{~s}^{-1}$ and $C_{A, 2}=798 \mathrm{1} \mathrm{s}^{-1}$, the

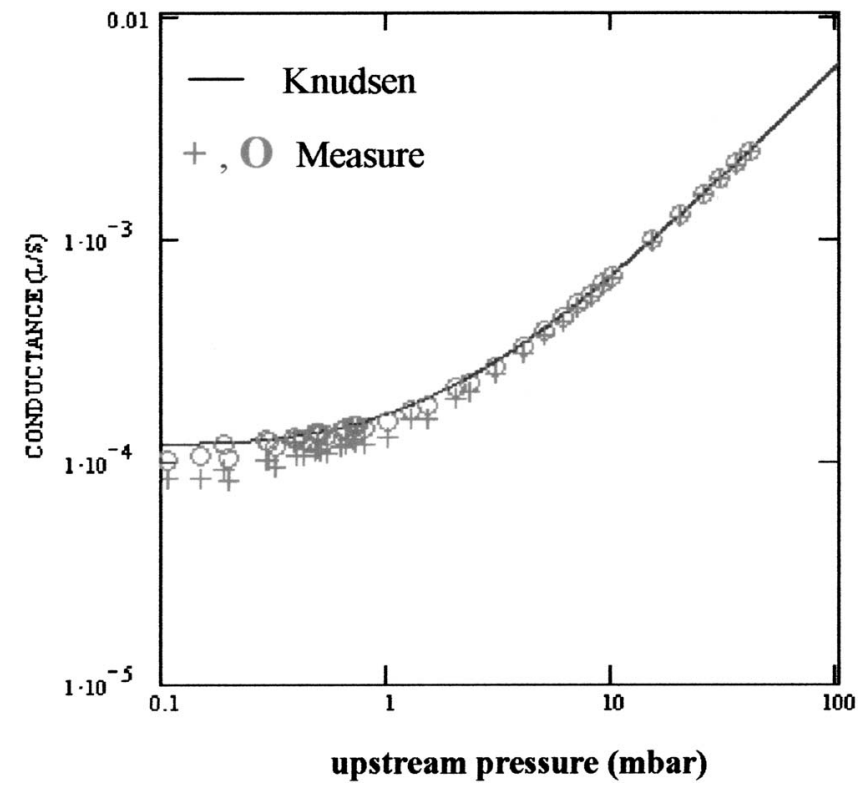

FIG. 4. The conductance $C 1$ as a function of the upstream pressure.

pressure $P_{0}$ in the volume of introduction will have to be higher than 1 mbar. $C_{R, 1}$ and $C_{A, 2}$ are calculated by using the Knudsen equation and with a correction factor $k$, respectively, of 5.3 and 4.5 (see Fig. 2). Before the nitrogen introduction, the system pressure is about $5 \times 10^{-7} \mathrm{~Pa}$. Table I recapitulates the measurements taken with and without the presence of the prepumping elbow. The principal measurement error comes from the difficulty in maintaining $P_{0}$ constant, and consequently the accuracy measurements of pressure for the spinning rotor gauges are estimated to within $\pm 5 \%$.

The first two lines of the Table I are values with the presence of the prepumping elbow, CC, and the remaining lines with the direct connection of the turbo pump. The measurement of these values was taken at a temperature of $25^{\circ} \mathrm{C}$. The difference in the value of the intrinsic conductance of cone $C_{R, 1}$ with and without the prepumping elbow is relatively small. The modification of the emission law of the molecules from the turbo-pump seems to influence only moderately the value of $C_{R, 1}$. The variation between the calculated conductance $\left(35.3 \mathrm{l} \mathrm{s}^{-1}\right)$ and measured one $\left(69 \mathrm{l} \mathrm{s}^{-1}\right)$

TABLE I. "Back" cone measurement.

\begin{tabular}{ccccc}
\hline \hline $\begin{array}{c}P_{0} \\
(\mathrm{mbar})\end{array}$ & $\begin{array}{c}C_{1} \\
\left(1 \mathrm{~s}^{-1}\right)\end{array}$ & $\begin{array}{c}P_{\mathrm{JAB} 1} \\
(\mathrm{mbar})\end{array}$ & $\begin{array}{c}P_{\mathrm{JAB} 2} \\
(\mathrm{mbar})\end{array}$ & $\begin{array}{c}C_{R, 1}=P_{0} C_{1} / \Delta P_{\mathrm{JAB}} \\
\left(1 \mathrm{~s}^{-1}\right) \pm 12 \%\end{array}$ \\
\hline 4 & $3.16 \times 10^{-4}$ & $4.79 \times 10^{-5}$ & $2.96 \times 10^{-5}$ & 69.1 \\
10 & $7 \times 10^{-4}$ & $2.59 \times 10^{-4}$ & $1.6 \times 10^{-4}$ & 70.7 \\
& & & 66.7 \\
1 & $1.6 \times 10^{-4}$ & $5.4 \times 10^{-6}$ & $3 \times 10^{-6}$ & 66.9 \\
5 & $3.8 \times 10^{-4}$ & $6.8 \times 10^{-5}$ & $3.96 \times 10^{-5}$ & 65.4 \\
10 & $7 \times 10^{-4}$ & $2.4 \times 10^{-4}$ & $1.39 \times 10^{-4}$ & 66.5 \\
15 & $9.5 \times 10^{-4}$ & $4.9 \times 10^{-4}$ & $2.76 \times 10^{-4}$ & 62.4 \\
20 & $1.23 \times 10^{-4}$ & $9.06 \times 10^{-4}$ & $5.12 \times 10^{-4}$ & 64.3 \\
30 & $1.8 \times 10^{-4}$ & $1.96 \times 10^{-4}$ & $1.12 \times 10^{-4}$ & \\
\hline \hline
\end{tabular}


(a)
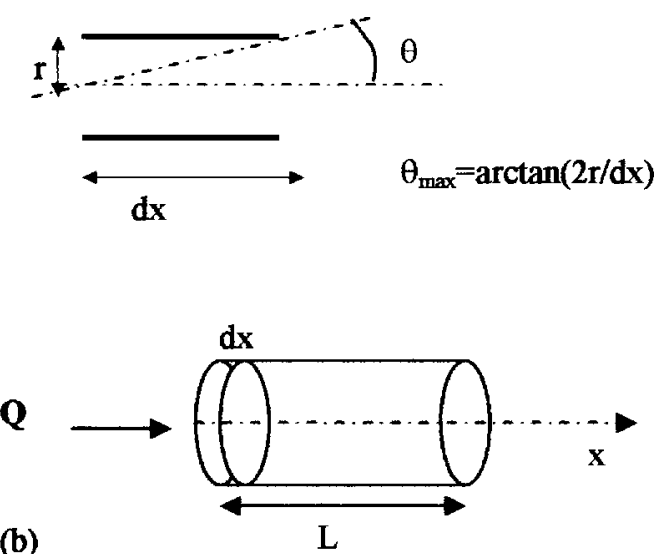

FIG. 5. Cylindrical tube scheme (a) on a surface element $d s$; (b) on all surface tube.

is explained by the fact that first one is calculated compared to the pressures upstream and downstream (entry and exit surfaces) while the second one is measured compared to the tube surface. Moreover, in our case, it is necessary to take into account the position of the gauge JAB1, between the two cones.

\section{CALCULATION OF THE SURFACE DISTRIBUTION OF PRESSURE}

\section{A. The case of the cylindrical tube}

Initially, to calculate the collision number on a surface element, $d s$, one uses the Clausing $P_{\mathrm{Cl}}$ probability of the number of molecules crossing without collision.

$$
P_{c l}=\int_{0}^{\theta_{\max }} 2 \cos (\theta) \sin (\theta) S(\theta) d \theta
$$

with $S(\theta)$ the transmission probability of the molecules without collision for a given angle $\theta$ [Fig. 5(a)].

$$
\begin{gathered}
S(\theta)=\frac{2}{\pi}\left[\arccos (y)-y\left(1-y^{2}\right)^{1 / 2}\right] \\
\text { with } y=[L /(2 r)] \tan (\theta),
\end{gathered}
$$

thus for a length $L \rightarrow d x$ and a cosine distribution law of the molecules, the number of collisions for $N$ emitted molecules is

$$
N_{\text {choc }}=N\left(1-P_{c l}\right)=N\left(\frac{d x}{r}\right) .
$$

To determine the surface pressure at the beginning of a circular conductance, it is possible to use the Oatley method, by associating a tube of length $d x$ and of transmission probability $W_{1}=N_{\text {choc }} / 2$, to a second length $L$ of probability $W_{2}$ [see Fig. 5(b)].

At each passage, whatever the direction, one assumes that the number of hits on the surface $2 \pi r d x$ is $N d x / r$. It is obvious that this is only an approximation, not taking into account the "beam" effect, with variations of molecular spatial distributions for different crossings. In addition, in order to

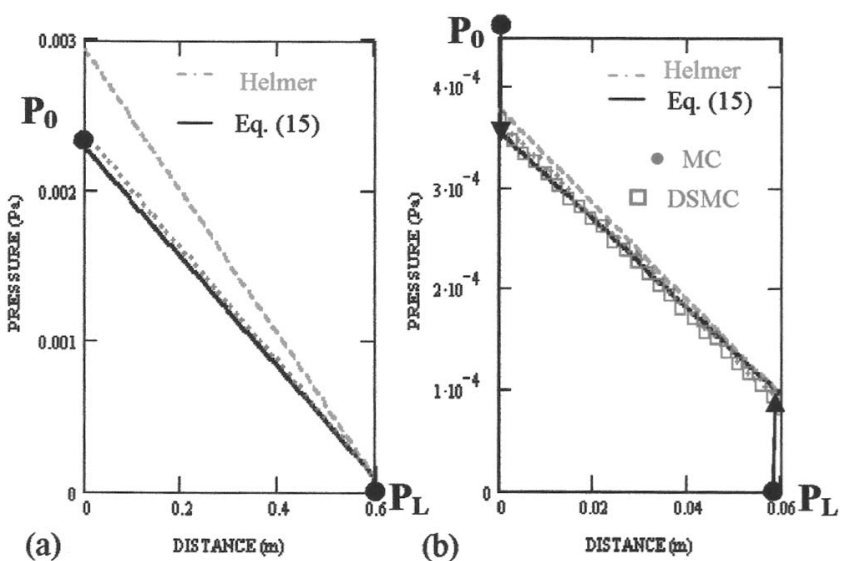

FIG. 6. Surface pressure distribution for cylindrical conductance for nitrogen at $20^{\circ} \mathrm{C}$, with a flux of $2.8 \times 10^{-5} \mathrm{~Pa} \mathrm{~m}^{3} \mathrm{~s}^{-1}$, for total absorption (a) for $L / r=30$ with $r=2 \mathrm{~cm}$; (b) for $L / r=3$ with $r=2 \mathrm{~cm}$

be able to simulate a flow, the rejected molecules are injected into the circuit, at the entrance of $W_{1}$. By assuming that $W_{1}$ is close to 1 , we obtain

$$
N_{\text {choc }}=N \frac{d x}{r}\left(\frac{2-W_{2}}{W_{2}}\right) \text {. }
$$

Consequently, the surface pressure for $d x \rightarrow 0$ can be expressed by

$$
P_{S_{0}}=\frac{Q}{(1 / 4) V_{m} \pi r^{2}}\left(\frac{2-W_{2}}{2 W_{2}}\right) .
$$

By deriving the $Q=\delta C d P$ equation (with $\delta C$ the intrinsic conductance for a length $d x$ ) and while taking as initial condition $P_{s_{0}}$ (obtained by the Oatley method), the surface pressure distribution, for a steady flow $Q$ and for a perfect pump (sticking coefficient $=1$ ), we have

$$
\begin{aligned}
P_{S}(x) & =\frac{-Q}{C_{L} L} x+P_{S_{0}} \\
& =-\frac{Q}{\frac{1}{4} V_{m} \pi r^{2} W_{2}}\left[\frac{1}{2}+\left(1-W_{2}\right)\left(\frac{1}{2}-\frac{x}{L}\right)\right] .
\end{aligned}
$$

Helmer and Levi ${ }^{9}$ obtained the same result by using a differential form of the Oatley equation and by taking $1 / W_{2}=1$ $+1 / f$ with $f=2 r / L$ as form factor. Figure 6 compares the pressure distribution calculated by Eq. (15), by the HelmerLevi method, and also by the Monte Carlo method (MC or DSMC), for tubes of radius $0.02 \mathrm{~m}$ and length-radius ratios of 3 and 30 . The flow $Q$ is $2.8 \times 10^{-5} \mathrm{~Pa} \mathrm{~m}^{3} \mathrm{~s}^{-1}$ for nitrogen at $20{ }^{\circ} \mathrm{C}$. The transmission probabilities $W_{2}$ are, respectively, 0.421 and 0.0797 . For the short tube, we observe a good correlation between the $P_{0}\left[=Q /\left(C_{e} W_{2}\right)\right]$ of the upstream chamber and surface pressure $P_{s_{0}}$. According to Eq. (15), this difference is constant and independent of $W_{2}, \Delta P=P_{0}-P_{s_{0}}$ $=Q /\left(2 C_{e}\right)$ and it also applies to the difference in pressure downstream $P_{\mathrm{SL}}-P_{L}$ (in our case $P_{L}=0$ ). This variation of pressure between volume and surface seems to be a good 

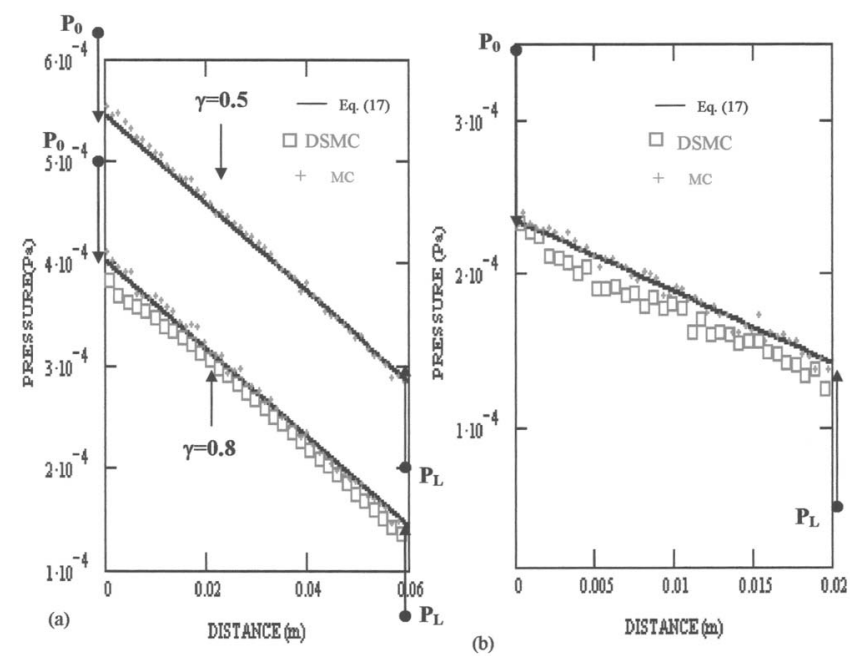

FIG. 7. Surface pressure distribution for cylindrical conductance for nitrogen at $20{ }^{\circ} \mathrm{C}$, with a flux of $2.8 \times 10^{-5} \mathrm{~Pa} \mathrm{~m}^{3} \mathrm{~s}^{-1}$, (a) for different sticking coefficients $\gamma=0.8$ and 0.5 and for $L / r=3$ with $r=2 \mathrm{~cm}\left(W_{2}=0.421\right)$; (b) for a sticking coefficient $\gamma=0.8$ and for $L / r=1$ with $r=2 \mathrm{~cm}\left(W_{2}=0.672\right)$.

approximation in spite of the fact that the change of the molecular spatial distribution is not taken into account. For the long tube, the $\Delta P$ becomes negligible with respect to $P_{0}$ $\left(\Delta P / P_{0}=W_{2} / 2\right.$ with small $\left.W_{2}\right)$, and the difference with the Helmer-Levi method arises from the fact that, according to a number of authors, the form factor $f$ for a long tube tends towards $8 r /(3 L)$.

It is possible to extend the method of Oatley in order to take into account a pumping capacity $S_{0}=C_{e} \gamma$, with $C_{e}$ the entrance conductance corresponding to the inlet pump and $\gamma$ the sticking coefficient on this surface. The collision number on the element $d x$ becomes

$$
N_{\text {choc }}=N \frac{d x}{r}\left[\frac{2-W_{2}}{W_{2}}+\frac{2(1-\gamma)}{\gamma}\right] .
$$

With the same reasoning as previously, the surface distribution of pressure is expressed by

$$
P_{S}(x)=\frac{Q}{C_{e} W_{2}}\left[\frac{1}{2}+\left(1-W_{2}\right)\left(\frac{1}{2}-\frac{x}{L}\right)+\frac{W_{2}(1-\gamma)}{\gamma}\right] .
$$

The upstream and downstream pressures are expressed classically by, respectively, $P_{0}=Q /\left(C_{e} W_{2}\right)\left[1+W_{2}(1-\gamma) / \gamma\right]$ and $P_{L}=\left(Q / C_{e}\right)[(1-\gamma) / \gamma]$ with $P_{0}-P_{L}=Q /\left(C_{e} W_{2}\right)$. Figure 7 represents the pressure distribution on the tube surface for the $L / r$ ratio and for different sticking coefficients. Equation (17) gives, to a good approximation, the pressure measured by a gauge for a system of pumping connected by a tube to a large chamber. It should be noted that the difference between the surface pressures and the upstream and downstream pressures is constant as previously whatever the tube length and the pumping speed. It is equal to $\Delta P=P_{0}-P_{s_{0}}$ $=P_{\mathrm{SL}}-P_{L}=Q /\left(2 C_{e}\right)$.

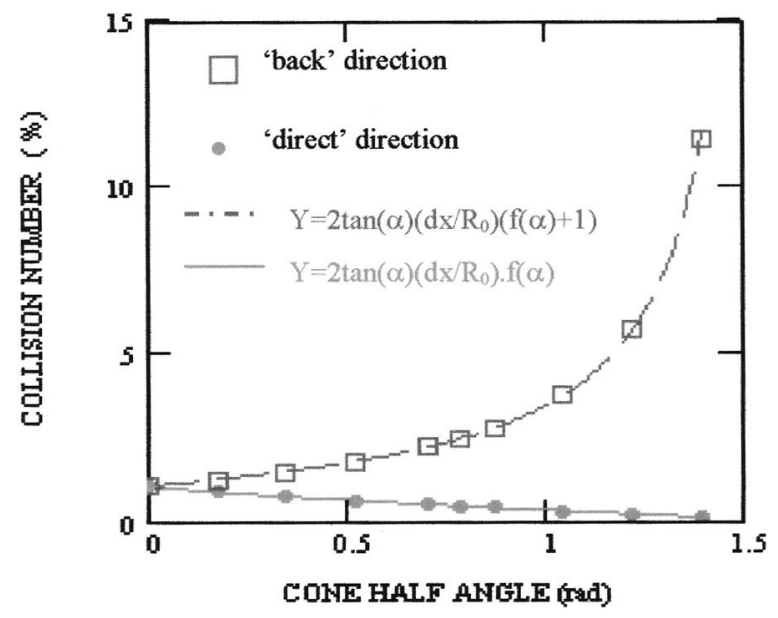

FIG. 8. Collision number on the cone surface for $L \rightarrow d x\left(10^{-5} \mathrm{~m}\right)$ and for radius $R_{0}=0.01 \mathrm{~m}$ as a function of half-angle of cone.

\section{B. The case of a conical tube}

In the same way, to calculate the collision number on an surface element $d s$, one uses the probability of Clausing, $P_{\text {cone }}$, of the number of molecules crossing without collision.

$$
\begin{aligned}
P_{\text {cone }}= & \int_{0}^{\theta_{\max }} 2 \cos (\theta) \sin (\theta) S_{c}(\theta) d \theta \\
& \text { with } \theta_{\max }=\arctan \left[\left(R_{k}-R_{0}\right) / L\right],
\end{aligned}
$$

with $S_{c}(\theta)$ the transmission probability of the molecules without collision for a given angle $\theta$ and the direct direction of the cone.

$$
\begin{aligned}
S_{c}(\theta)= & \frac{1}{\pi}\left\{\arccos (y)-y\left(1-y^{2}\right)^{1 / 2}\right. \\
& \left.+\frac{R_{K}^{2}}{R_{0}^{2}}\left[\arccos \left(y_{1}\right)-y_{1}\left(1-y_{1}^{2}\right)^{1 / 2}\right]\right\},
\end{aligned}
$$

with $y=\left[L /\left(2 R_{0}\right)\right] \tan (\theta)+\left(R_{0}^{2}-R_{k}^{2}\right) /\left[2 L R_{0} \tan (\theta)\right]$ and $y 1$ $=\left[L /\left(2 R_{k}\right)\right] \tan (\theta)+\left(R_{k}^{2}-R_{0}^{2}\right) /\left[2 L R_{k} \tan (\theta)\right]$. For $\theta$ ranging between 0 and $\alpha, S_{c}(\theta)=1$. This equation can be solved numerically. For a length $L \rightarrow d x$, a cosine distribution law for the collision number for $N$ emitted molecules is $N_{\text {choc }}=N(1$ $\left.-P_{\text {cone }}\right)$. According to Fig. 8, we can express this collision number as a function of the cone half-angle, $\alpha$.

$$
\begin{aligned}
N_{\text {choc }}= & N 2 \frac{d x}{R_{0}} \tan (\alpha) f(\alpha) \\
& \text { with } f(\alpha)=\frac{1}{2 \tan (\alpha)}\left(1-\frac{8}{3} \frac{\alpha}{\pi}+\frac{4}{3} \frac{\alpha^{2}}{\pi}\right) .
\end{aligned}
$$

With the same reasoning, the collision number for a cone in the back direction is

$$
N_{\text {choc }-r}=N 2 \frac{d x}{R_{0}} \tan (\alpha)[f(\alpha)+1] .
$$

To determine the surface pressure, in the same way as that for a tube, it is possible to extend the Oatley method again, 


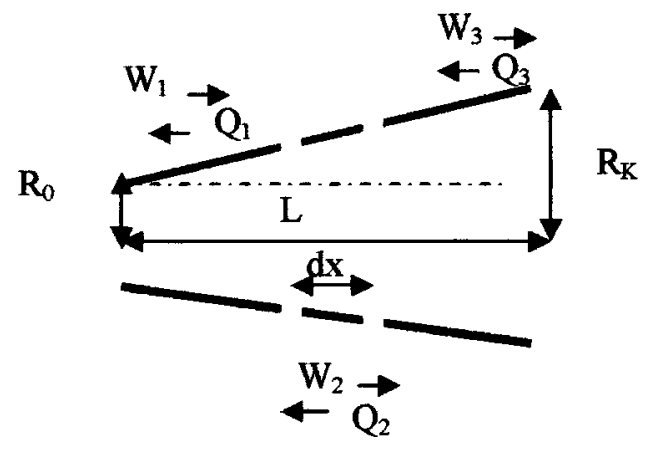

by associating several cones of transmission probability in the direct $W$ and back $Q$ directions [see Fig. 9]. For each passage of molecules, one enters the collision number on the surface of the length element $d x$ while applying, according to the direction, Eq. (19) or (20). The probabilities $W_{2}$ and $Q_{2}$ tend towards 1 and by simulating a flow and a capacity of pumping, we get a total collision number according to $x$, for an element $d x$,

FIG. 9. Addition of several cones with different transmission probabilities.

$$
N_{\text {choc }}=N \frac{2 d x \tan (\alpha)}{R(x)}\{\underbrace{\left.f(\alpha)\left(\frac{2-W_{3}}{W_{3}}\right)+\left(\frac{1-W_{3}}{W_{3}}\right)+\frac{Q_{3}}{W_{3}} \frac{(1-\gamma)[2 f(\alpha)+1]}{\gamma}\right\}}_{h},
$$

with $Q_{3} / W_{3}=R^{2}(x) / R_{k}^{2}, W_{3}$ is of course a function of $x$ and can be expressed on the basis of Eqs. (6) and (7) by

$$
\frac{1}{W_{3}}=1+\frac{\left[R(x)+R_{k}\right]}{\frac{16}{3} R_{k}^{2} k[L-x / R(x)]}(L-x)
$$

$$
\text { and } R(x)=R_{0}+x \tan (\alpha) \text {, }
$$

the factor $k$ varies slightly according to $L / R_{0}$ (see Fig. 2), for an angle of $40^{\circ}, k$ varies from 4.5 to 5.3 for, respectively, a $L / R_{0}=1$ and $L / R_{0}=10$. We can roughly express this variation as an equation. For a direct cone of $40^{\circ}, k\left(t=L / R_{0}\right)$ $\approx(4.5+2.65 t+2.72 t 2) /(1+0.56 t+0.5 t 2)$.

\section{Calculation of the surface distribution by the method known as the extension of Oatley}

By estimating that the number of shocks per unit time on the surface of the cone length $d x$ is $\Gamma=(1 / 4) V_{m} n S(d x)$ (with $n$ molecular density), the surface distribution of pressure is expressed as

$$
P_{S_{1}}(x) \approx \frac{Q N_{\text {choc }}}{(1 / 4) V_{m} S(d x) N}=\frac{Q \sin (\alpha)}{(1 / 4) V_{m} \pi R(x)^{2}} h .
$$

\section{Calculation of the surface distribution by the traditional method (Knudsen)}

As in the case of the tube, by deriving the $Q=\delta C d P$ equation (with $\delta C$ intrinsic cone conductance given by Knudsen and for a length $d x$ ) and while taking $P_{s_{1}}(0)$ as initial condition (obtained by the Oatley method), this distribution of surface pressure can be written as follows:

$$
\begin{aligned}
P_{S_{2}}(x) & =\int \frac{-Q}{(2 / 3) V_{m} \pi k} \frac{d x}{R(x)^{3}} \\
& =\frac{Q}{(4 / 3) V_{m} \pi k \tan (\alpha)}\left[\frac{1}{R(x)^{2}}-\frac{1}{R_{0}^{2}}\right]+P_{S_{1}}(0) .
\end{aligned}
$$

\section{Extension of the Oatley method for a cone in the back direction}

In the same manner, we can determine the surface pressure distribution for a cone in the back direction, with, in this case, $R(x)=R_{k}-x \tan (\alpha)$, a corrective factor $k^{\prime}=k /[1$ $+(16 / 3) k \tan (\alpha)]$ and with a collision number,

$$
\begin{aligned}
N_{\text {choc }-R}= & N \frac{2 d x \tan (\alpha)}{R(x)}\left\{f(\alpha)\left(\frac{1-Q_{3}}{Q_{3}}\right)+\left[\frac{1+f(\alpha)}{Q_{3}}\right]\right. \\
& \left.+\frac{W_{3}}{Q_{3}} \frac{(1-\gamma)(2-f(\alpha)+1)}{\gamma}\right\} .
\end{aligned}
$$

It is obvious that this is only an approximation which does not take into account the beam effects (the changes of the spatial distribution of the molecules for the different passages). According to Fig. 10, one can note that Eq. (23) derived from the conductance calculated by Knudsen is not valid, or only for one correction factor $k$ equal to 1 .

In this case, as in the extension of the Oatley method, we obtain a relatively good approximation of the surface pressure compared to simulation by Monte Carlo. This approximation improves when the pumping speed decreases. Figure 10 shows the influence of a pump on the surface pressure distribution at the cone exit. This pump will modify the 


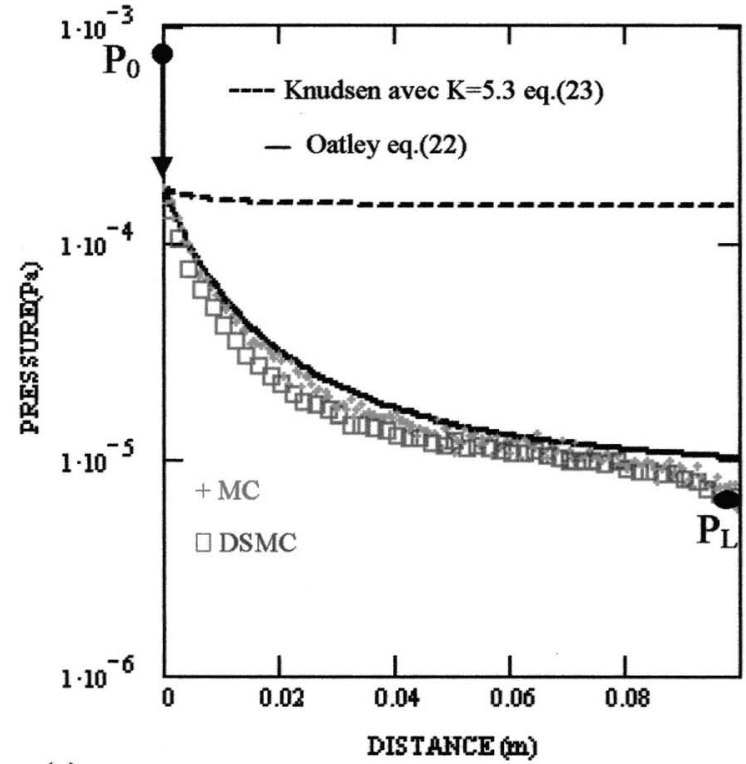

(a)

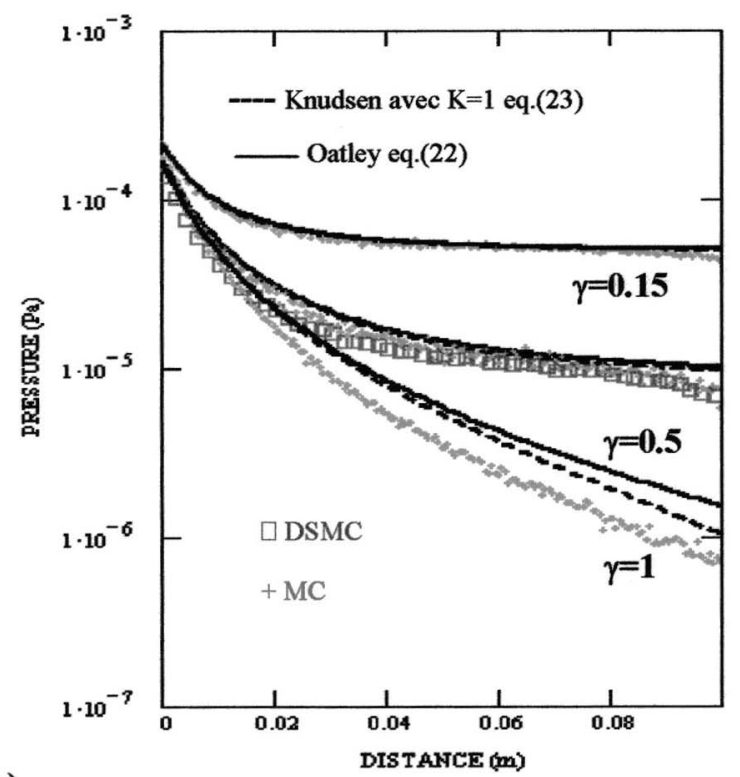

(b)

FIG. 10. Surface pressure distribution for conical conductance of "direct" direction for nitrogen at $20{ }^{\circ} \mathrm{C}$, with a flux $2.8 \times 10^{-5} \mathrm{~Pa} \mathrm{~m}^{3} \mathrm{~s}^{-1}$, for different sticking coefficients, for $L / R_{0}=10$ with $r=1 \mathrm{~cm}$ and $\alpha=40.28^{\circ}$.

spatial distribution of the molecules at the cone exit. In the case of the large sticking coefficient, the spatial distribution of the molecules at the cone exit is not Lambert's law. For the back cone, the approximation by the extension of the Oatley method is satisfactory and that for all pumping speeds (Fig. 11). The good agreement between these two methods encourages to consider the spatial distribution on the exit tube as Lambert's law. The Knudsen equation is not very sensitive to the correction factor $k$ and greatly diverges when approaching downstream opening and for high pumping capacities.

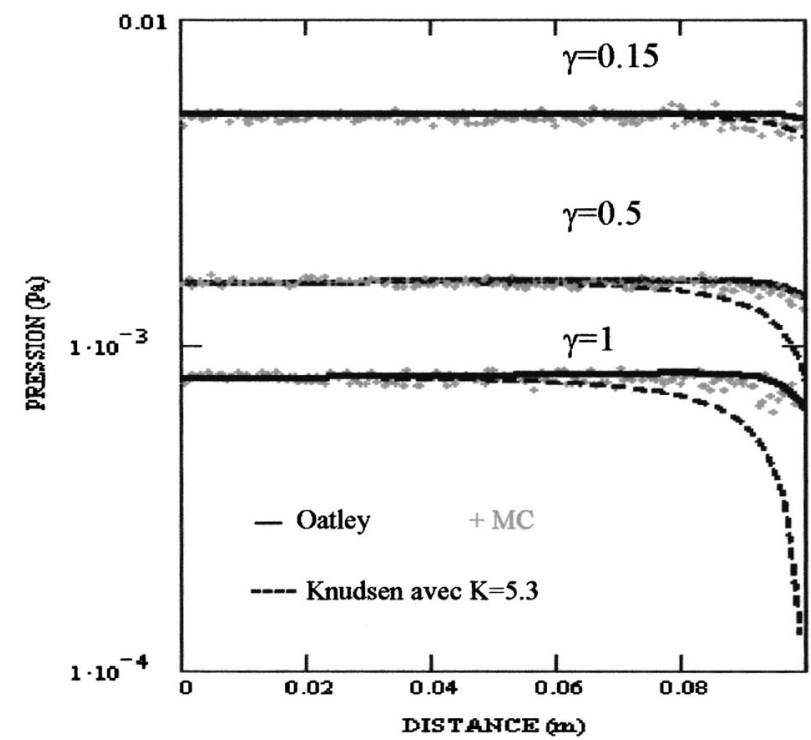

FIG. 11. Surface pressure distribution for conical conductance of "back" direction for nitrogen at $20^{\circ} \mathrm{C}$, with a flux $2.8 \times 10^{-5} \mathrm{~Pa} \mathrm{~m}^{3} \mathrm{~s}^{-1}$, for different sticking coefficients, for $L / R_{0}=10$ with $r=1 \mathrm{~cm}$ and $\alpha=40.28^{\circ}$.

\section{COMPARISON BETWEEN MEASUREMENT AND SIMULATIONS}

The experimental setup (see Fig. 3) is formed by the junction of a back cone and a direct cone. It is necessary for the extension of the Oatley method to determine the sticking coefficients corresponding to a turbo pumping speed of $S_{0}=125 \mathrm{l} \mathrm{s}^{-1}$, for the second cone $\gamma=S_{0} / C_{e 2}=0.135$ and for the first cone $\gamma 1=0.768$ first [with $C_{A, 2}=7981 \mathrm{~s}^{-1}$ for $k$ $=4.8$ and $\left.1 /\left(C_{e 1} \gamma 1\right)=1 / 125-1 / C_{e 2}+1 / C_{A, 2}+1 / C_{e 1}\right]$. By the Knudsen method, it is enough to fix the pressure at the entry of the first cone or the exit of the second with

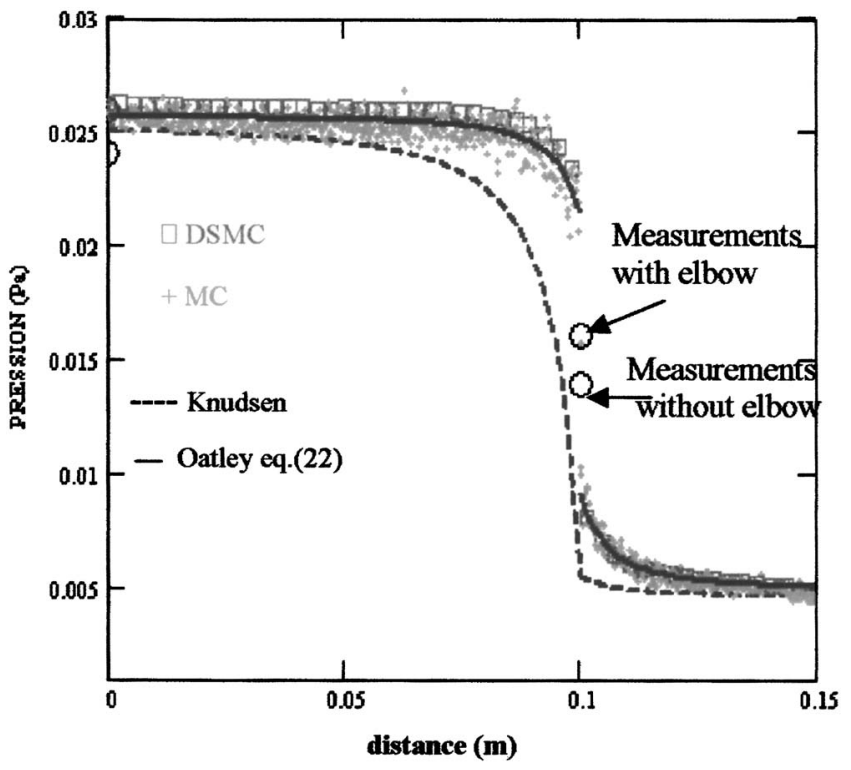

FIG. 12. Surface pressure distribution on the two conical tubes of experimental device for a pumping speed of $1251 / \mathrm{s}$. 
$P_{1} \approx Q /\left(1 / S_{0}-1 / C_{e 2}+1 / C_{A, 2}+1 / C_{R, 1}\right) \quad$ or $\quad P_{0}=Q /\left(1 / S_{0}\right.$ $-1 / C_{e 2}$ ). For simulations by Monte Carlo, we simulate the two cones and as well as the turbo pump at the system exit as a unique system and we take as sticking coefficient of $\gamma=0.135$.

Figure 12 shows a good correspondence between simulation by Monte Carlo and Oatley. A small variation is noted with the points of measurements taken without a prepumping elbow but remains within the error margin. The pressure recorded by the two spinning rotor gauges seems to validate in our case the simulations methods and consequently to confirm the correction factors $k$.

However, the formula for the surface pressure distribution derived from the Knudsen conductance with the adequate $k$ factors diverges to give a pressure at $x=0.1 \mathrm{~m}$ of 5.5 $\times 10^{-3} \mathrm{~Pa}$ instead of a measured pressure of $1.4 \times 10^{-2} \mathrm{~Pa}$ and a simulated pressure of $1.55 \times 10^{-2} \mathrm{~Pa}$.

\section{CONCLUSION}

The Monte Carlo method made it possible to determine the correction factor $k$ to apply to the Knudsen conductance formula in the case of the cone. In addition, the extension of the Oatley method gives a good approximation of the surface pressure distribution for tubes or cones taking into account a pump at the exit of the conductance. This distribution is all the more sensitive since the lengths are short and the pumping capacities are important. This can, therefore, have a great influence on the vacuum metrology. Our measurements seem to support our simulations but they need to be improved and extended to other geometries.

\section{ACKNOWLEDGMENT}

The author would like to thank Dr. T. Garvey for improving the English of the manuscript.

${ }^{1}$ M. Smoluchowski, Foundation of Vacuum Science and Technology, edited by J. M. Lafferty (Wiley, New York, 1998), p. 87.

${ }^{2}$ R. P. Henry, Cours de Sciences et Technique du vide (SFV, Paris, 1965), Tome 2, p. 41.

${ }^{3}$ P. Clausing, J. Vac. Sci. Technol. 8, 636 (1971).

${ }^{4}$ J. Gomez-Goni and P. J. Lobo, J. Vac. Sci. Technol. A 21, 1452 (2003).

${ }^{5}$ P. Swemin and M. Niewinski, Vacuum 67, 359 (2002).

${ }^{6}$ R. P. Iczkowski, J. L. Margrave, and S. M. Robinson, J. Phys. Chem. 67, 229 (1963).

${ }^{7}$ A. Pace, CERN PS/ML/Note 87-3 (1987).

${ }^{8}$ G. A. Bird, Molecular Gas Dynamics and the Direct Simulation of Gas Flows (Clarendon, Oxford, 1994).

${ }^{9}$ J. C. Helmer and G. Levi, J. Vac. Sci. Technol. A 20, 1216 (2002). 